\title{
Thrombocytosis is linked to increased cancer risk, study finds
}

This Research News article by Zosia Kmietowicz contains a misprint (BMJ 2017;357:j2481, doi:10.1136/bmj.j2481, 23 May 2017). In the fifth paragraph, the number of women with thrombocytosis who developed cancer in the next 12 months was 1355 (not 355). The positive predictive value of $6.2 \%$, however, was correct. 